\title{
Ablative Volume of Radiofrequency Ablation Related to Intrahepatic Recurrence-Free Survival of Hepatocellular Carcinoma
}

\author{
Nakarin Inmutto ${ }^{1}$ Siripong Thaimai ${ }^{1}{ }^{\circledR}$ Tanop Srisuwan ${ }^{1}{ }^{\circledR}$ Thanate Kattipathanapong ${ }^{1}{ }^{\circledR}$ \\ Natthaphong Nimitrungtawee ${ }^{10}$ \\ ${ }^{1}$ Department of Radiology, Faculty of Medicine, Chiang Mai \\ University, Chiang Mai, Thailand \\ Arab J Intervent Radiol 2021;5:76-81. \\ Address for correspondence Nakarin Inmutto, MD, Department of \\ Radiology, Faculty of Medicine, Chiang Mai University, Chiang Mai, \\ Thailand 50200 \\ (e-mail: nakarin.i@cmu.ac.th; ninmuttomd@gmail.com).
}

\begin{abstract}
Keywords

- hepatocellular carcinoma

- Radiofrequency ablation

- recurrence

Purpose The aim of this study was to evaluate intrahepatic recurrence-free survival of hepatocellular carcinoma (HCC) after Radiofrequency ablation (RFA)

Methods A retrospective cohort study of single HCC treated by RFA between 2015 and 2017. Fifty patients were enrolled in the study. Tumor volume and ablative volume were measured by using Syngo.via application (Siemen Healthineers, United States). Ablative volume classified into small and large ablative volumes. Ablative margin was evaluated by visual comparison between pre- and post-RFA images and classified into two groups: closed ablative margin $(<0.5 \mathrm{~cm})$ and large ablative margin $(>0.5 \mathrm{~cm})$. The recurrence tumor was classified as local tumor progression (LTP) and intrahepatic distant recurrence (IDR). The recurrence-free survival rate and independent risk for tumor recurrence were analyzed.

Results Recurrence-free survival rate at the first, second, third, and fourth year after RFA was $83,56,44$, and $44 \%$, respectively. The average recurrence-free survival time in large ablative volume group was significantly longer than small ablative volume group (31.57 vs. 8.50 months, $p=0.003$ ). A significant independent risk factor for tumor recurrence was large ablative volume (hazard ratio $=0.12,95 \%$ confidence interval $=0.02-0.84, p=0.033)$. The IDR group had ratio of actual ablative volume by ideal ablative volume (ablative ratio) higher than LTP group and nonrecurrent group.

Conclusion The large ablative volume prevented tumor recurrence and increased recurrence-free survival rate. But aggressive ablation with high ablative volume ratio could increase risk of IDR.
\end{abstract}

\section{Introduction}

Percutaneous Radiofrequency ablation (RFA) or microwave ablation (MWA) has an important role in treatment early hepatocellular carcinoma (HCC) size less than $3 \mathrm{~cm}$ because of its effectiveness, feasibility, and less complications. ${ }^{1,2}$ There are two types of intrahepatic recurrence found in patients with HCC after RFA, including local tumor published online February 14, 2022
DOI https://doi.org/ $10.1055 / \mathrm{s}-0042-1742654$. ISSN 2542-7075. (c) 2022. The Pan Arab Interventional Radiology Society. All rights reserved.

This is an open access article published by Thieme under the terms of the Creative Commons Attribution-NonDerivative-NonCommercial-License, permitting copying and reproduction so long as the original work is given appropriate credit. Contents may not be used for commercial purposes, or adapted, remixed, transformed or built upon. (https://creativecommons.org/ licenses/by-nc-nd/4.0/)

Thieme Medical and Scientific Publishers Pvt. Ltd., A-12, 2nd Floor, Sector 2, Noida-201301 UP, India 
progression (LTP) and intrahepatic distant recurrence (IDR). LTP occurs along the peripheral margin of the low attenuated ablation zone, while IDR is a new HCC tumor remote from the margin of the original ablation zone.

Although these ablation therapies can achieve complete necrosis of small HCC, recurrence is still common. The 3-year LTP rates for patients treated by RFA with an ablation margin of 0.5 to $1.0 \mathrm{~cm}$ are reportedly 10 to $20 \%$. The risk factors for tumor recurrence after RFA included tumor size, located near the main portal branch or inferior vena cava, elevated $\alpha$ fetoprotein level, platelet count, and antihepatitis C antibody positivity. $^{3-7}$

Several studies believe tumor hypoxia and hepatic parenchymal hypoperfusion after RFA are significant predictive factors of tumor recurrence. After RFA, the transition zone between normal liver tissues and ablation zone exposes residual cancer cells to a hypoxic microenvironment. The hypoxia-inducible factors (HIF) are active via hypoxic signaling pathway. It causes tumor cells to become more invasive, metastatic, chemoresistant resulting to intrahepatic recurrent. ${ }^{8,9}$ Lee et al reported that hepatic parenchymal hypoperfusion caused by either ischemia resulting from portal vein injury or congestion from hepatic vein damage after RFA is a significant predictive factor of recurrence after RFA of a single nodular HCC. ${ }^{10}$

We propose that large ablative volume might be associated with tumor hypoxia and hepatic parenchymal hypoperfusion. It may induce vascular endothelial growth factor and cause intrahepatic tumor recurrence. The aim of this study was to compare recurrence-free survival between patients who had large ablative volume and small ablative volume.

\section{Materials and Methods}

\section{Diagnosis of HCC}

According to Liver Reporting \& Data System (LI-RADS) and American Association for the Study of Liver Diseases guideline, patients with liver mass compatible with LI-RADS 4 and LI-RADS 5 were enrolled in this study.

\section{Study Design and Patient Population}

Patients with HCC treated with RFA between 2015 and 2017 was 283 visits. The inclusion criteria were as follows: patients with LI-RADS 4 or 5 , single lesion, size less than $3.0 \mathrm{~cm}$, Barcelona Clinic Liver Cancer stage 0 or A, patients should have a complete response at the first follow-up images at 4 to 8 months and underwent follow-up computed tomography (CT) every 3 to 6 months. We excluded patients with a history of previous treatment of HCC and the presence of other malignancies. Finally, 50 patients were included.

This retrospective cohort study was approved by the institute research committee and informed consent was waived (Approval no. RAD-2562-0649).

\section{RFA Protocol}

RFA was performed by using ultrasound-guided percutaneous puncture with the multitined expandable electrode (LeVeen Needle Electrodes; Boston Scientific Cooperation,
United States). We used 1-cm stepwise expansion of the tines and double ablation method until the tines were fully expanded. ${ }^{11}$ All patients underwent follow-up CT scans or magnetic resonance imaging (MRI) 4 weeks after RFA. The goal of the treatment was to achieve complete ablation in the tumor ablation zones, which were the hypoattenuating unenhanced areas in the arterial and the portal venous phases that were larger than the previous tumor.

\section{Measurement Technique and Parameters}

The size of the lesion was measured at the greatest diameter and calculated the tumor volume by using Syngo.via application (Siemens Healthineers, United States) in arterial phase. The ablation zone was measured at the greatest diameter and calculated the ablative volume by using Syngo.via application in portovenous phase.

Ablative margin was classified into two groups: closed ablative margin (margin $<0.5 \mathrm{~cm}$ ) and large ablative margin (margin $>0.5 \mathrm{~cm}$ ). To define the ablative margin as accurate as possible, we performed qualitative side-by-side comparison of CT scans obtained before and after RF ablation by a radiologist with 12 years of experience. The adjacent hepatic vessels or the hepatic capsule were used to facilitate comparison. ${ }^{12}$

The ideal ablative volume was calculated by $4 / 3 \times \pi \times$ (D/ $2+0.5)^{3}$ under the assumption of spherical shape and $0.5-$ $\mathrm{cm}$ safety ablative margin. ${ }^{13,14}$ When ablative volume was anywhere larger than ideal ablative volume, we defined as large ablative volume. While ablative volume was smaller than ideal ablative volume, we defined as small ablative volume. The ratio between ablative volume and ideal ablative volume was defined as ablative volume ratio.

All patients underwent follow-up CT every 3 to 6 months. The recurrent date was defined as the date that CT or MRI demonstrated tumor recurrence. Recurrent was classified as LTP or IDR. The endpoint of nonrecurrent group used the last CT or MRI date in Picture Archiving and Communication System.

\section{Statistical Analyses}

Statistical analyses were proceeded by SPSS software (version 24) and Stata (version 15.1). Descriptive data with normal distribution are reported as mean \pm standard deviation (SD). The difference between two groups was compared using Student's $t$-test or the Mann-Whitney test. The cumulative recurrent rate during the follow-up was recorded. Recurrence-free survival curve was estimated by the Kaplan-Meier method and statistically significant was compared with the Log-Rank test. Multivariate analysis using the stepwise cox proportional hazard model was performed for the variable with $p<0.10$ in the univariate analysis to investigate independent risk factors for tumor recurrence.

\section{Results}

Patients' population, tumor, and ablative parameters were shown in - Table 1. There was no significant difference in 
78 Ablative Volume of RFA Related to IDR-Free Survival of HCC Inmutto et al.

Table 1 Baseline characteristics of all patients, patients with recurrence, patient without recurrence, LTP, and IDR

\begin{tabular}{|c|c|c|c|c|}
\hline & $\begin{array}{l}\text { All } \\
(n=50)\end{array}$ & $\begin{array}{l}\text { Recurrent } \\
(n=22)\end{array}$ & $\begin{array}{l}\text { Nonrecurrent } \\
(n=28)\end{array}$ & p-Values \\
\hline Age $(y)^{a}$ & $60.6 \pm 10.3$ & $58.82 \pm 10.19$ & $61.93 \pm 10.34$ & \\
\hline \multicolumn{5}{|l|}{ Sex (\%) } \\
\hline Male & $38(76)$ & $18(36)$ & $20(40)$ & \multirow[t]{2}{*}{0.512} \\
\hline Female & $12(24)$ & $4(8)$ & $8(16)$ & \\
\hline \multicolumn{5}{|l|}{ BCLC stage (\%) } \\
\hline 0 & $31(62)$ & $13(26)$ & $18(36)$ & \multirow[t]{2}{*}{0.774} \\
\hline$A$ & $19(38)$ & $9(18)$ & $10(20)$ & \\
\hline \multicolumn{5}{|l|}{ Child-Pugh (\%) } \\
\hline A & $40(80)$ & $17(34)$ & $23(46)$ & \multirow[t]{2}{*}{0.732} \\
\hline $\mathrm{B}$ & $10(20)$ & $5(10)$ & $5(10)$ & \\
\hline Hepatitis B (\%) & $26(52)$ & $13(26)$ & $13(26)$ & 0.407 \\
\hline Hepatitis C (\%) & $18(36)$ & $9(18)$ & $9(18)$ & 0.565 \\
\hline Tumor size $(\mathrm{cm})^{\mathrm{a}}$ & $1.80 \pm 0.56$ & $1.87 \pm 0.56$ & $1.75 \pm 0.58$ & 0.493 \\
\hline Tumor volume $\left(\mathrm{cm}^{3}\right)^{\mathrm{a}}$ & $3.25 \pm 2.72$ & $3.84 \pm 2.99$ & $2.81 \pm 2.47$ & 0.192 \\
\hline Ablation size $(\mathrm{cm})^{\mathrm{a}}$ & $2.99 \pm 2.33$ & $3.32 \pm 3.45$ & $2.72 \pm 3.33$ & 0.599 \\
\hline Ablation volume $\left(\mathrm{cm}^{3}\right)^{\mathrm{a}}$ & $12.22 \pm 8.40$ & $12.36 \pm 7.69$ & $12.11 \pm 9.06$ & 0.250 \\
\hline Ablation margin $(\mathrm{cm})^{\mathrm{a}}$ & $0.36 \pm 0.35$ & $0.36 \pm 0.37$ & $0.37 \pm 0.34$ & 0.005 \\
\hline $\begin{array}{l}\text { Ablation margin more } \\
\text { than } 0.5 \mathrm{~cm}(\%)\end{array}$ & $19(38)$ & $8(16)$ & $11(22)$ & 1.00 \\
\hline $\begin{array}{l}\text { Ablation volume more } \\
\text { than ideal ablation volume (\%) }\end{array}$ & $47(94)$ & $20(40)$ & $27(54)$ & 0.576 \\
\hline $\begin{array}{l}\text { Ablation volume/ } \\
\text { Ideal ablation volume ratio }\end{array}$ & $5.98 \pm 8.13$ & $7.10 \pm 11.24$ & $5.10 \pm 4.46$ & 0.547 \\
\hline
\end{tabular}

Abbreviation: BCLC, Barcelona Clinic Liver Cancer; IDR, intrahepatic distant recurrence; LTP, local tumor progression; SD, standard deviation.

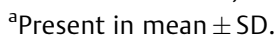

baseline characteristics between the recurrence and nonrecurrence groups. The follow-up ranged from 1 to 48 months. Twenty-two patients developed tumor recurrence after treatment including 6 LTP and 16 IDR. The recurrence-free survival rate in first, second, third, and fourth years after RFA were $83,56,44$, and $44 \%$, respectively. The recurrence-free survival curve was shown in -Fig. 1.

The recurrence-free survival time in large ablative margin was slightly longer than closed ablative margin
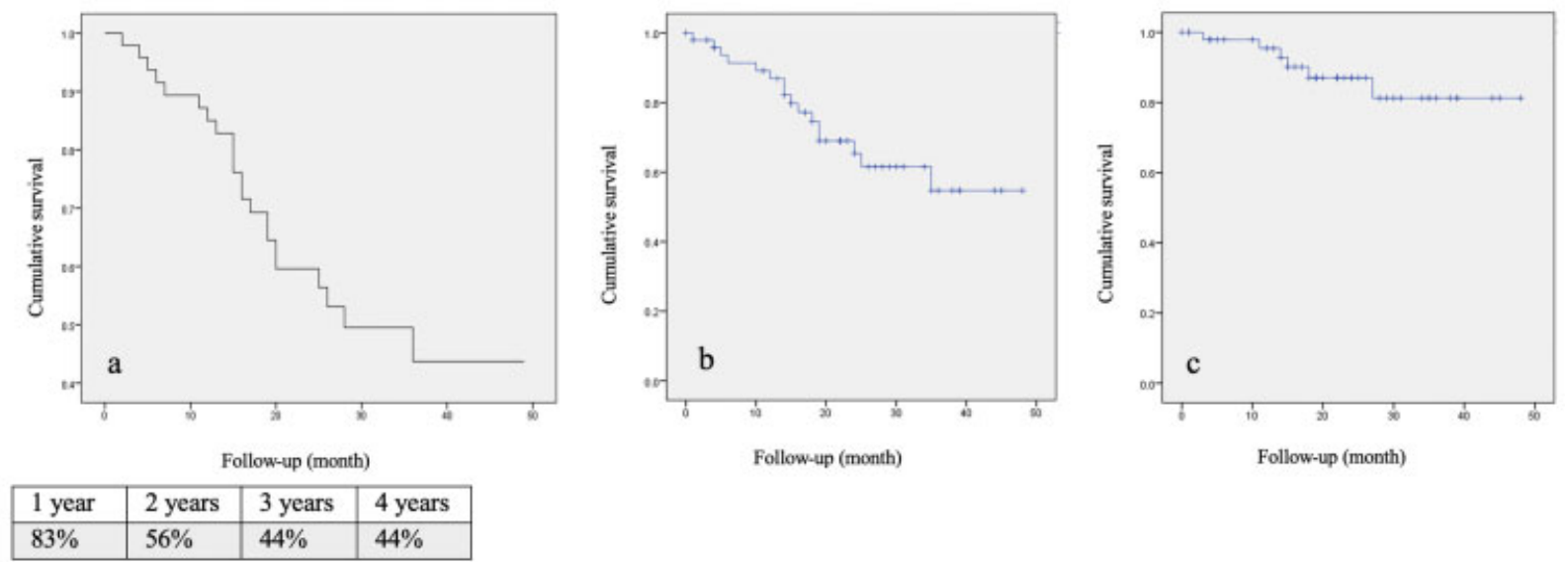

\begin{tabular}{|l|l|l|l|}
\hline 1 year & 2 years & 3 years & 4 years \\
\hline $83 \%$ & $56 \%$ & $44 \%$ & $44 \%$ \\
\hline
\end{tabular}

Fig. 1 Recurrence-free survival curve in patients underwent radiofrequency ablation, in all patients (A), local tumor progression, (B) and intrahepatic distant recurrence (C). 

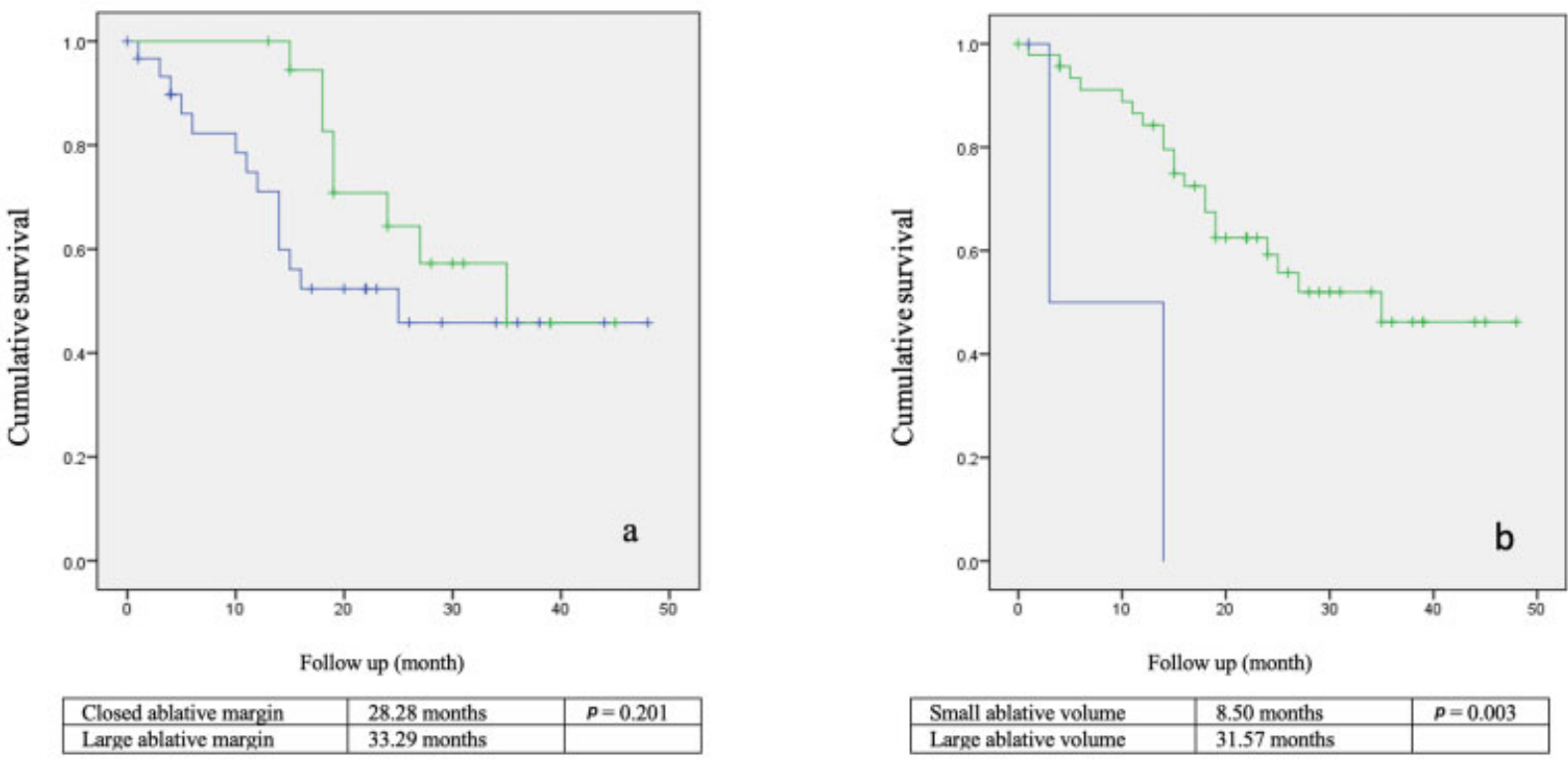

Fig. 2 (A) Comparison of recurrence-free survival between closed ablative margin (blue) and large ablative margin (green), which shows average 28.28 and 33.29 months, respectively. (B) Comparison of recurrence-free survival between small ablative volume (blue) and large ablative volume (green), which shows average 8.50 and 31.57 months, respectively.

(33.29 vs. 28.28 months, $p=0.201$ ). Patients with large ablative volume showed a significantly longer recurrence-free survival time than small ablative volume (31.57 vs. 8.50 months, $p=0.003$; - Fig. 2).
In the univariate analysis, the potential factors for tumor recurrence after RFA were $\alpha$-fetoprotein level, tumor size, tumor volume, and large ablative volume (-Table 2 ). These four factors were further analyzed with multivariate

Table 2 Logistic regression of the risk factor associated overall tumor recurrence

\begin{tabular}{|c|c|c|c|c|}
\hline \multirow[t]{2}{*}{ Variable } & \multicolumn{2}{|l|}{ Univariate analysis } & \multicolumn{2}{|c|}{ Multivariate analysis } \\
\hline & HR (95\%Cl) & $p$-Value & HR (95\%Cl) & $p$-Value \\
\hline Age $(y)$ & $0.98(0.95-1.02)$ & 0.543 & & \\
\hline Sex (female/male) & $0.68(0.23-2.02)$ & 0.494 & & \\
\hline BCLC stage $(0 / A)$ & $1.60(0.68-3.77)$ & 0.274 & & \\
\hline Child-Pugh (A/B) & $0.97(0.35-2.64)$ & 0.960 & & \\
\hline Albumin level & $0.92(0.53-1.62)$ & 0.790 & & \\
\hline AFP level & $1.00(1.00-1.00)$ & 0.010 & $1.00(0.99-1.01)$ & 0.099 \\
\hline LI-RADS (5/4) & $1.41(0.41-4.78)$ & 0.578 & & \\
\hline Tumor size $(\mathrm{cm})$ & $2.29(0.93-5.63)$ & 0.070 & $0.67(0.10-4.70)$ & 0.691 \\
\hline Tumor volume $\left(\mathrm{cm}^{3}\right)$ & $1.31(1.10-1.56)$ & 0.002 & $1.288(0.86-1.93)$ & 0.219 \\
\hline Arterial enhancement (N/Y) & $1.89(0.25-14.14)$ & 0.533 & & \\
\hline Portal washout (N/Y) & $1.71(0.22-12.92)$ & 0.602 & & \\
\hline Capsule enhancement (N/Y) & $0.88(0.38-2.03)$ & 0.761 & & \\
\hline Ablation time (min) & $0.99(0.95-1.04)$ & 0.909 & & \\
\hline Ablation size $(\mathrm{cm})$ & $1.06(1.93-1.21)$ & 0.342 & & \\
\hline Ablation volume $\left(\mathrm{cm}^{3}\right)$ & $0.99(0.95-1.04)$ & 0.891 & & \\
\hline Ablation margin (cm) & $0.51(0.15-1.65)$ & 0.263 & & \\
\hline Ablation margin more than $0.5 \mathrm{~cm}(\mathrm{~N} / \mathrm{Y})$ & $0.57(0.24-1.37)$ & 0.209 & & \\
\hline Ablation volume more than ideal ablation volume $(\mathrm{N} / \mathrm{Y})$ & $0.14(0.03-0.64)$ & 0.011 & $0.12(0.02-0.84)$ & 0.033 \\
\hline Ablation volume /Ideal ablation volume ratio & $1.00(0.95-1.05)$ & 0.853 & & \\
\hline
\end{tabular}

Abbreviations: AFP, $\alpha$-fetoprotein; BCLC, Barcelona Clinic Liver Cancer; Cl, confidence interval; HR, hazard ratio; LI-RADS, Liver Imaging Reporting and Data System. 
analysis. Only large ablative volume was an independent risk factor for tumor recurrence after RFA (hazard ratio [HR]= $0.12,95 \%$ confidence interval $[\mathrm{CI}]=0.02-0.84, p=0.033)$.

The univariate and multivariate analysis for LTP and IDR were shown (-Table 3 ).

Independent risk factors of LTP were ablation size (HR $=1.24,95 \% \mathrm{CI}=1.04-1.48, p=0.017)$ and large ablative volume $(\mathrm{HR}=0.007,95 \% \mathrm{CI}=0.0-0.124, p=0.001)$.

An independent risk factor of IDR was tumor volume (HR $=1.24,95 \% \mathrm{CI}=1.00-1.54, p=0.045)$. The HR for large ablative volume cannot be analyzed because all of the patients with IDR had large ablative volume.

\section{Discussion}

The overall recurrence-free survival rate in our study was similar to the prior study, which shows a recurrence-free survival rate at first and third years $\sim 74$ and $40 \%$, respectively. $^{15}$

Several studies tried to describe the risk factor for tumor recurrence especially ablative margin. The ablative margin less than $0.5 \mathrm{~cm}$ has a higher risk for tumor recurrence after RFA. ${ }^{13}$ In our study, we had a higher recurrence-free survival rate in large ablative margin than closed ablative margin, but it is not significant $(p=0.201)$. Furthermore, we found some patients had HCC adjacent to the liver capsule or vascular structures. These patients were described as a $0-\mathrm{mm}$ ablative margin, but the tumor recurrence did not occur. So, the ablative margin was not the good parameter to predict the recurrence-free survival rate. Ablative volume may be another parameter use to predict tumor recurrence. We found that patients with large ablative volume had a significantly higher recurrence-free survival rate than small ablative volume $(p=0.03)$.

Large ablative volume may produce hypoxic microenvironment and causes tumor recurrence. Lee et al proposed hepatic parenchymal hypoperfusion after RFA as a significant predictive factor of recurrence. ${ }^{10}$ We believe larger ablative volume could induce more HIF and play a role to develop intrahepatic tumor recurrence.

To balance between recurrent from inadequate margin and hypoxic microenvironment, we focus on ablative volume ratio instead of ablative volume. From our data, 94\% of patients had ablative volume more than ideal ablation volume. The average ablative volume was six times greater than ideal ablative volume, ablative volume ratio. Patient who had tumor recurrence had ablative ratio larger than the nonrecurrent group. The IDR patients had a higher ablation ratio; up to 8.6 time. Therefore, we presume the ablation volume ratio was the other predictive factor for tumor recurrent, especially IDR.

There are several limitations of this study. First, it was retrospective that caused variety of biases. Therefore, further studies are required in a prospective trial. Second, the measurement of ablation margin by side-by-side comparison technique by one radiologist may not be consistent. Test of observer agreement such as interobserver reliability by two radiologists should be analyzed in the further studies.

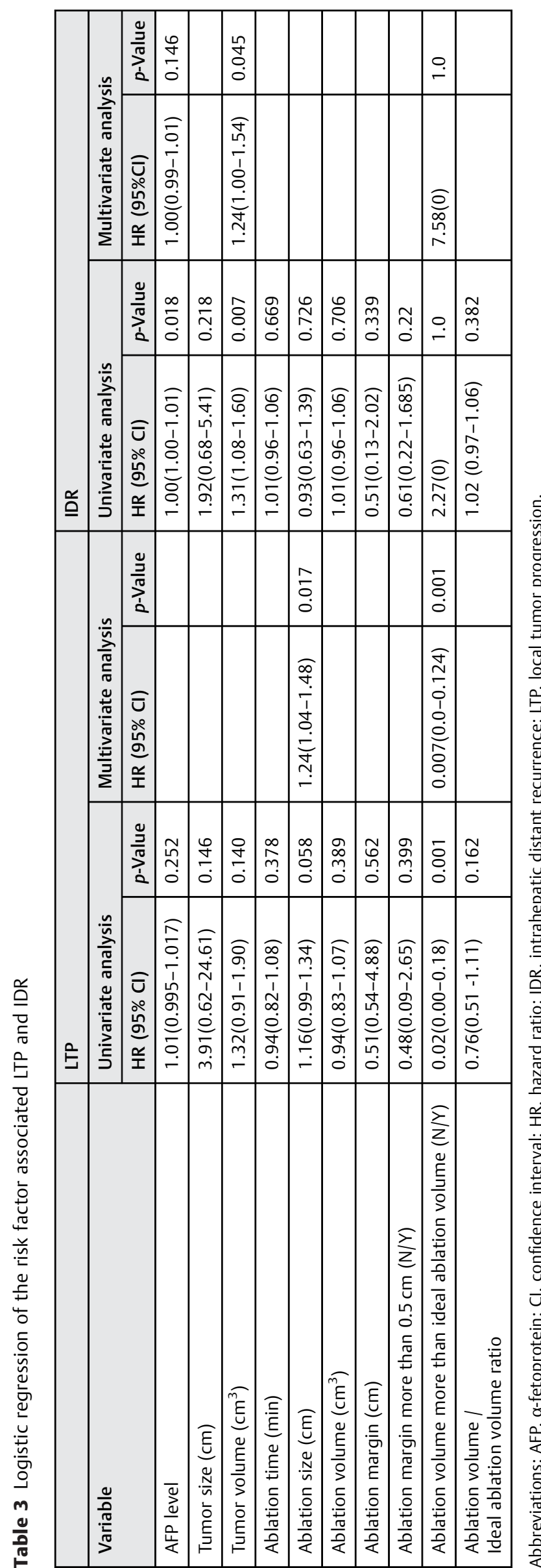

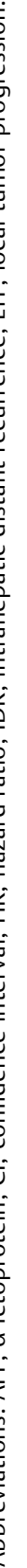


Additional, in some previous studies, they try to use intelligent software for fused pre- and post-treatment imaging. ${ }^{13,16}$ It had more accuracy for evaluating the ablation margin than manual registration, but it was not available in our department.

We concluded that a large ablative volume was the independent factor for predicting higher intrahepatic recurrence-free survival and decreased risk of LTP. Conversely, a large ablative volume with high ablative ratio might increase risk of IDR.

Previous Presentation

Poster presentation, CIRSE 2020 Summit, September 12th to 15 th, Virtual conference.

\section{Conflict of Interest}

This study was not supported by any funding.

The authors declare that they have no conflict of interest. For this type of study formal consent is not required.

Consent for publication was obtained for every individual person's data included in the study.

Large ablative volume of Radiofrequency ablation related to intrahepatic recurrence-free survival of hepatocellular carcinoma.

\section{References}

1 Kang TW, Kim JM, Rhim H, et al. Small hepatocellular carcinoma: radiofrequency ablation versus nonanatomic resection-propensity score analyses of long-term outcomes. Radiology 2015;275 (03):908-919

2 Koh PS, Chan AC, Cheung TT, et al. Efficacy of radiofrequency ablation compared with transarterial chemoembolization for the treatment of recurrent hepatocellular carcinoma: a comparative survival analysis. HPB (Oxford) 2016;18(01):72-78

3 Nicoli N, Casaril A, Abu Hilal M, et al. A case of rapid intrahepatic dissemination of hepatocellular carcinoma after radiofrequency thermal ablation. Am J Surg 2004;188(02):165-167

4 Kang TW, Lim HK, Cha DI. Aggressive tumor recurrence after radiofrequency ablation for hepatocellular carcinoma. Clin Mol Hepatol 2017;23(01):95-101
5 Angonese C, Baldan A, Cillo U, et al. Complications of radiofrequency thermal ablation in hepatocellular carcinoma: what about "explosive" spread? Gut 2006;55(03):435-436

6 Tateishi R, Shiina S, Akahane M, et al. Frequency, risk factors and survival associated with an intrasubsegmental recurrence after radiofrequency ablation for hepatocellular carcinoma. PLoS One 2013;8(04):e59040

7 Ruzzenente A, Manzoni GD, Molfetta M, et al. Rapid progression of hepatocellular carcinoma after radiofrequency ablation. World J Gastroenterol 2004;10(08):1137-1140

8 Tong Y, Yang H, Xu X, et al. Effect of a hypoxic microenvironment after radiofrequency ablation on residual hepatocellular cell migration and invasion. Cancer Sci 2017;108(04):753-762

9 Chen C, Lou T. Hypoxia inducible factors in hepatocellular carcinoma. Oncotarget 2017;8(28):46691-46703

10 Lee DH, Lee JM, Yoon JH, Kim YJ, Han JK. Thermal injury-induced hepatic parenchymal hypoperfusion: risk of hepatocellular carcinoma recurrence after radiofrequency ablation. Radiology 2017; 282(03):880-891

11 Inmutto N, Srisuwan T, Kattipatanapong T, Kaoworakarn C. Complete ablation rate of percutaneous radiofrequency ablation of hepatocellular carcinoma in favorable and unfavorable locations: using real time ultrasound guidance and expandable electrode needle. Chiang Mai Med J 2018;57(01):1-9

12 Ke S, Ding XM, Qian XJ, et al. Radiofrequency ablation of hepatocellular carcinoma sized $>3$ and $\leq 5 \mathrm{~cm}$ : is ablative margin of more than $1 \mathrm{~cm}$ justified? World J Gastroenterol 2013;19(42):7389-7398

13 Jiang C, Liu B, Chen S, Peng Z, Xie X, Kuang M. Safety margin after radiofrequency ablation of hepatocellular carcinoma: precise assessment with a three-dimensional reconstruction technique using CT imaging. International journal of hyperthermia: the official journal of European Society for Hyperthermic Oncology. North Am Hyperthermia Group 2018;34(08):1135-1141

14 Nakazawa T, Kokubu S, Shibuya A, et al. Radiofrequency ablation of hepatocellular carcinoma: correlation between local tumor progression after ablation and ablative margin. AJR Am J Roentgenol 2007;188(02):480-488

15 Hong SN, Lee SY, Choi MS, et al. Comparing the outcomes of radiofrequency ablation and surgery in patients with a single small hepatocellular carcinoma and well-preserved hepatic function. J Clin Gastroenterol 2005;39(03):247-252

16 Takeyama N, Mizobuchi N, Sakaki M, et al. Evaluation of hepatocellular carcinoma ablative margins using fused pre- and postablation hepatobiliary phase images. Abdom Radiol (NY) 2019;44 (03):923-935 